\title{
Phase-field study of pore-grain boundary interaction
}

\author{
Johannes HÖTZER, ${ }^{*, *, \dagger}$ Veronika REHN, ${ }^{*}$ Wolfgang RHEINHEIMER, ${ }^{*}$ \\ Michael J. HOFFMANN* and Britta NESTLER**
}

*Institute for Applied Materials (IAM), Karlsruhe Institute of Technology (KIT), Haid-und-Neu-Str. 7, 76131 Karlsruhe, Germany **Institute of Materials and Processes, Karlsruhe University of Applied Sciences, Moltkestrasse 30, D 76133 Karlsruhe, Germany

\begin{abstract}
During final stage sintering, a complex interplay of densification and grain growth dominates microstructural evolution. Grain growth starts, when pore drag effects become less important due to pore shrinkage. This grain growth then decreases the driving force available for sintering. Accordingly, the interplay of pores and grain boundaries needs to be considered in detail. A phasefield model was extended to treat pore dynamics under consideration of pressure stability. To study pore attachment and detachment at moving interfaces, an idealized hexagonal microstructure with a constant driving force relationship for pore migration is constructed. Additionally, realistic polycrystalline microstructures were used. The model is in good agreement with experiments and analytic equations. Three different cases were observed in the realistic microstructure: pore attachment at the moving interface, partial and total pore detachment. However, in the partial case, the initial location of pores was found to be important: pores tend to migrate from quadruple junctions over triple junctions to grain boundary planes, where they eventually detach. This results in a variation of pore detachment, which is not captured in analytic equations. Therefore large simulation setups are required to reflect the impact of initial pore location on pore drag effects.
\end{abstract}

(C2016 The Ceramic Society of Japan. All rights reserved.

Key-words : Final stage sintering, Pore drag, Pore attachment, Pore detachment, Phase-field simulations

\section{Introduction}

The understanding of solid-state sintering of ceramic materials is crucial for their technical application, since for each case a tailored microstructure is required, i.e. size and shape of grains and pores. While in some cases a defined porous structure is needed, most applications require dense polycrystalline materials. Additionally to the density, the grain size needs to be considered: most applications require a fine-grained microstructure.

Sintering and grain growth are two different processes and base on different driving forces. Sintering is driven by surface energy and particle size. An increasing particle size reduces the driving force for sintering. Thus sintering and grain growth are conflictive processes: typically, a high density in sintering can be obtained for materials with little grain growth [Fig. 1(a)], while pronounced grain growth during sintering results in remaining porosity in the microstructure [Fig. 1(b)].

In general, solid-state sintering can be divided into three stages, namely initial, intermediate and final stage. During the initial stage, necks are formed between powder particles without significant densification. In the intermediate stage the microstructure evolves into a three-dimensional network of particles with pore channels in between. Most of the densification occurs in this stage. As sintering continues, in the final stage the pore channels break up into isolated pores at junctions.

Coarsening becomes important in final stage sintering. This is caused by a change in the interaction of pores and grain boundaries: in the intermediate stage, large pores can exert strong dragging forces to the boundary motion (pore drag). In final stage, pores become smaller during densification. At a given pore size

\footnotetext{
Corresponding author: J. Hötzer; E-mail: johannes.hoetzer@kit. edu

* Preface for this article: Dol http://dx.doi.org/10.2109/jcersj2.124.P4-1
}

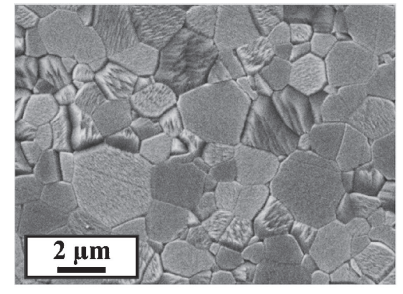

(a)

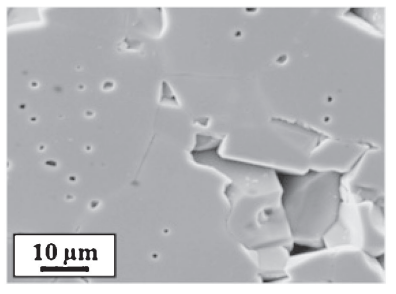

(b)
Fig. 1. $\mathrm{SrTiO}_{3}$ sintered at $1425^{\circ} \mathrm{C}$ for $4 \mathrm{~h}$ (a) and $\mathrm{KNN}$ (PotassiumSodium-Niobate) sintered at $1105^{\circ} \mathrm{C}$ for $2 \mathrm{~h}$ (b). ${ }^{1)}$ In the former, almost full density is reached with very little grain growth. In the latter case, strong grain growth prevented sintering in the final stage and a porosity remains in the microstructure.

the driving force on grain boundaries may exceed the decreasing drag effects of the pores and grain growth occurs. This decreases the available driving force for sintering. Accordingly, two phenomena can be observed: (i) detachment of pores from the grain boundary and (ii) attachment of pores to the moving grain boundary. The details of these phenomena are very important in sintering, since pore attachment retards or even inhibits grain growth and preserves a high driving force for complete densification.

Different theoretical considerations on concurrent densification and grain growth based on a mean field approach can be found in the literature. ${ }^{2), 3)}$ E.g. Brook assumed an idealized microstructure and determined the transition from pore-controlled to boundarycontrolled grain growth as a function of grain growth and pore size. ${ }^{2)}$ Bennison and Harmer ${ }^{4}$ took conditions for pore separation into account. Gottstein and Shvindlerman ${ }^{5)}$ presented a theoretical model of grain boundary migration in the presence of mobile particles. However, the impact of attached pores on grain growth is difficult to investigate during sintering and specific setups are 
needed. Therefore, Rödel and Glaeser ${ }^{6}$ developed an experimental setup to study pore-boundary interactions and pore drag, free from the influence of densification.

So far, analytic models based on mean field approaches are not capable of reflecting sintering and grain growth under consideration of pore drag in a sufficient way. Through technological progress in computer engineering, ${ }^{7)}$ numerical simulations of complex sintering processes became possible. Thus, several studies focused on modelling the sintering process with computer simulations. Tikare et al. ${ }^{8)}$ presented a three-dimensional MonteCarlo Potts model to simulate grain growth in the presence of mobile pores in an idealized hexagonal microstructure. They captured effects such as reduced grain boundary velocity through attached pores as predicted by the analytics of Gottstein and Shvindlerman. ${ }^{5)}$ Another approach to study the sintering processes under consideration of local microstructure evolution is given by the phase-field method. Grain growth was studied by Selzer, ${ }^{9}$ Kumar et al. ${ }^{10)-12)}$ and Vondrous $^{13)}$ for different anisotropic models using phase-field simulations. Jing et al. ${ }^{14)}$ simulated the interplay of pores and grain boundaries under hydrostatic pressure. Wang et al. ${ }^{15}$ ) developed a phase-field model, that considers various physical processes such as grain growth and diffusion mechanisms to study the sintering process of spherical particles. Kumar et al. ${ }^{16)}$ used a slightly modified version to investigate the impact of unequal sized particles. Asp and Ågren ${ }^{17)}$ developed a vacancy driven phase-field approach to simulate the sintering process.

However, so far very little information is available on the impact of pores on grain boundary migration, although this is of high importance in microstructural evolution during final stage sintering. Therefore, we present a phase field approach to study pore drag effects (i.e. pore attachment and detachment) in wellcontrollable idealized and realistic microstructures.

\section{Model}

To study pore attachment and pore detachment at grain boundaries during final sintering stage, we use a general phasefield model based on the work of Nestler ${ }^{18)}$ under consideration of the thermodynamics of interfaces. The model is implemented in the highly parallel multi-physics framework $\mathrm{P}_{\mathrm{ACE}} 3 \mathrm{D} .{ }^{9), 19)-22)}$

\subsection{Order parameter}

We consider the $d$-dimensional domain $\Omega \subset \mathbb{R}^{d}$ with $d \in$ $\{1,2,3\}$. For $N \in \mathbb{N}$, we define the index sets $\alpha, \beta \in \mathbb{I}:=$ $[1, \ldots, N]$ to distinguish the different phase-fields. The phase-field $\phi_{\alpha}: \Omega \times \mathbb{R} \rightarrow[0,1]$ describes a continuous function $\phi_{\alpha}(x, t)$ of the local phase fraction at the spacial position $\boldsymbol{x} \in \Omega$ and the time $t \in \mathbb{R}$. The set of phase-field variables are defined as a vector on a simplex, $\phi \in \Delta^{N} \forall \boldsymbol{x}, t$. The simplex is defined as

$$
\Delta^{N}=\left\{v \in[0,1]^{N} \mid \sum_{\alpha=1}^{N} v_{\alpha}=1\right\} .
$$

The phase-fields represent the physical properties like thermodynamic phases and orientations, respectively.

The region in which $\phi_{\alpha}=1$ is called bulk $B_{\alpha}:=$ $\left\{\boldsymbol{x} \in \Omega \mid \phi_{\alpha}(\boldsymbol{x}, t)=1\right\}$. The diffuse regions between the disjoint bulks are called interface. The total interface region is defined as $I_{\Omega}:=\Omega \backslash \cup_{\alpha \in N} B_{\alpha}$. The interface between two phases $\phi_{\alpha}$ and $\phi_{\beta} \quad$ is $I_{\alpha \beta}:=\left\{\boldsymbol{x} \in I_{\Omega} \mid \phi_{\alpha}(\boldsymbol{x}, t)+\phi_{\beta}(\boldsymbol{x}, t)=1\right\}$. The region, in which one phase-field is present, is defined as $\Omega_{\alpha}=$ $I_{\alpha \beta} \bullet B_{\alpha}, \forall \beta \in N$. Accordingly the complementary region, in which the phase-field is not present is defined as $\Omega_{\alpha}^{\mathrm{C}}=\Omega \backslash \Omega_{\alpha}$.

\subsection{Phase-field model}

The free energy functional depending on the phase-fields $\phi$ is formulated as

$$
\mathcal{F}(\phi)=\int_{\Omega}\left[\varepsilon a(\phi, \nabla \phi)+\frac{1}{\varepsilon} \omega(\phi)\right]+\sum_{\alpha=0}^{N} f_{\alpha} h_{\alpha}(\phi) d \Omega
$$

The first part of the functional Eq. (2) consists of the gradient energy density $\alpha(\phi, \nabla \phi)$ and the interfacial energy density $\omega(\phi)$. The second part represents the bulk energy density $f_{\alpha}$ with the interpolation function $h_{\alpha}(\phi)$, for the phase $\alpha$. The parameter $\varepsilon$ is related to the interface thickness and is used to scale the diffuse interface thickness. In this work $\varepsilon$ is four, corresponding to the interface thickness of about ten cells.

In order to model a defined diffuse transition between two or more phases, a gradient energy density of the form

$$
a(\phi, \nabla \phi)=\sum_{\substack{\alpha, \beta=1 \\(\alpha<\beta)}}^{N, N} \gamma_{\alpha \beta}\left|\vec{q}_{\alpha \beta}\right|^{2}
$$

is used, with the interface energy $\gamma_{\alpha \beta}$ and the general gradient vector $\vec{q}_{\alpha \beta}=\phi_{\alpha} \nabla \phi_{\beta}-\phi_{\beta} \nabla \phi_{\alpha}$ orthogonal to the interface. The interfacial energy density is defined by a multi-obstacle potential of the form

$\omega(\phi)= \begin{cases}\frac{16}{\pi^{2}} \sum_{\substack{\alpha, \beta=1 \\(\alpha<\beta)}}^{N, N} \gamma_{\alpha \beta} \phi_{\alpha} \phi_{\beta}+\sum_{\substack{\alpha, \beta, \delta=1 \\(\alpha<\beta<\delta)}}^{N, N, N} \gamma_{\alpha \beta \delta} \phi_{\alpha} \phi_{\beta} \phi_{\delta}, & \phi \in \Delta^{N}, \\ \infty, & \text { else. }\end{cases}$

The higher order term $\gamma_{\alpha \beta \delta}$ in Eq. (4) is used to reduce the occurrence of third or higher order phases in the two phase interfaces. ${ }^{18), 23)}$ To model the pressure $p_{\alpha}^{t}$ in a pore of the phasefield $\alpha$ with the volume $V_{\alpha}^{t}=\int_{\Omega} \phi_{\alpha}(x, t) d \Omega \mid x \in \Omega_{\alpha}$ at time $t$, we assume an ideal gas. With the ideal gas law $p_{\alpha}^{t} V_{\alpha}^{t}=n_{\alpha} R T=$ const $\mid \forall t \in \mathbb{R}$ and the assumption of a constant temperature over the domain $\Omega$, the bulk energy density can be derived as

$$
f_{\alpha}=\frac{p_{\alpha}^{0} V_{\alpha}^{0}}{V_{\alpha}^{t}} .
$$

As interpolation function for the for the bulk energy density, we employ

$$
h_{\alpha}(\phi)=\frac{\phi_{\alpha}^{2}}{\sum_{\beta=1}^{N} \phi_{\beta}^{2}}
$$

proposed by N. Moelans in. ${ }^{24)}$ The expression fulfills the constraints of Eq. (1) for more than two phases.

To minimize the functional Eq. (2), we calculate the variational derivative and obtain the evolution equation of Allen-Cahn type

$$
\begin{aligned}
\tau \epsilon \frac{\partial \phi_{\alpha}}{\partial t}= & -\frac{\delta \mathcal{F}(\phi)}{\delta \phi_{\alpha}} \\
= & -\{\underbrace{\epsilon\left[\frac{\partial \alpha(\phi, \nabla \phi)}{\partial \phi_{\alpha}}-\nabla \cdot \frac{\partial \alpha(\phi, \nabla \phi)}{\partial \nabla \phi_{\alpha}}\right]}_{:=r h s_{\alpha}} \\
& \underbrace{\frac{1}{\epsilon} \frac{\partial \omega(\phi)}{\partial \phi_{\alpha}}+\frac{\partial f_{\alpha} h_{\alpha}(\phi)}{\partial \phi_{\alpha}}-\lambda}_{:=r h s_{\alpha}}\},
\end{aligned}
$$

with $\tau$ as a kinetic coefficient and the Lagrange multiplier 


$$
\lambda=\frac{1}{N} \sum_{\alpha=1}^{N} r h s_{\alpha}
$$

to ensure the constraint $\sum_{\alpha}^{N} \phi_{\alpha}=1$ from Eq. (1) and the pressure driving force $\partial f_{\alpha} h_{\alpha}(\phi) / \partial \phi_{\alpha}$. The kinetic coefficient of the interfaces is calculated as

$$
\tau=\sum_{\substack{\alpha, \beta=1 \\(\alpha<\beta)}}^{N, N} \tau_{\alpha \beta} \phi_{\alpha} \phi_{\beta}
$$

with $\tau_{\alpha \beta}$ as the reciprocal mobility.

For simulations with many phase-fields, the vector $\phi$ can be locally reduced without loss of generality. Kim et al. ${ }^{25}$ showed for a phase-field model of ideal grain growth, that in one point a maximum of six grains in 3D (five in 2D) can be in contact with each other. This allows to reduce the number of equations to solve, and hence, the computational time and the required memory. For arbitrary constant $\boldsymbol{x}, t$, the index set of active phases is defined as $\mathbb{A}:=\left\{\alpha \in \mathbb{I} \mid \nabla \phi_{\alpha}>0\right\}$. With this, the reduced phase-field vector with the size $L=|\mathbb{A}| \leq N$ of the active phases is defined as $\boldsymbol{\Phi}:=\left(\phi_{\alpha}\right)_{\alpha \in \mathbb{A}}$. It exists a map

$$
\begin{aligned}
M: \Delta^{N} & \rightarrow \Delta^{L} \\
\phi & \mapsto \Phi
\end{aligned}
$$

and its inverse

$$
\begin{aligned}
M^{-1}: \Delta^{L} & \rightarrow \Delta^{N} \\
\boldsymbol{\Phi} & \mapsto \phi
\end{aligned}
$$

in which all not contained indizes are zero $\phi:=\left(\phi_{\alpha}\right)_{\alpha \in \mathbb{I}}$, $\phi_{\alpha}=0 \forall \alpha \in \mathbb{I} \backslash \mathbb{A}$.

With this reduction of the phase-field vector length and the assumption of not more than six phase-fields being active in one cell, we can reduce the number of equations in Eq. (8) to a maximum of six. Therefore, the required calculation time and memory depends no longer on the number of phase-fields in the system. This method enables the calculation of large grain systems as required for this work.

\section{Results and discussion}

In final stage sintering, isolated pores form at the grain boundaries. Thereby, the gas inside the pore becomes pressurized and acts against the densification. This effect is studied in the first part of this section. During further grain growth, pores start to interact with grain boundaries. Subsequently we compare the velocity of pore-laden and pore-free boundaries for idealized settings. For a realistic setting, the pore detachment in dependence of the pore size and spacing as well as the grain size in polycrystals is studied.

All parts are structured as follows: first we depict the simulation geometry and introduce the analytic equations. Afterwards the simulation results are presented and discussed.

\subsection{Stability of pores in grains}

During detachment of pores from grain boundaries, pores become distorted and are finally trapped inside a grain. In this section, we study the stability of pores and validate the model by Comparison with physical properties, as e.g. the ideal gas law. We place a pore with a non-equilibrium pressure, diameter and interface energy inside a grain and simulate until the equilibrium state between the forces of curvature minimization and gas pressure is reached. In the equilibrium state the pressure resulting from Eq. (5) and the radii of the pore are measured. The pressure difference can then be calculated between the chosen initial pressure and the measured one. This study serves as validation case for the pressure model and as basis for the more complex pore grain boundary interactions. The pressure difference $\Delta p$ of a spherical pore inside a grain with the interface energy $\gamma_{\alpha \beta}$ can be calculated analytically by the Young-Laplace equation

$$
\Delta p=\frac{\gamma_{\alpha \beta}}{r_{p}^{\text {equ. }}}(d-1)
$$

with $d$ as the dimension of the considered domain and $r_{p}^{\text {equ. }}$ the equilibrium radius of the pore.

To validate the model extension, the results of two and three dimensional simulations are evaluated, if the pressure reaches equilibrium according to the Young-Laplace equation. As initial pressure, the value of $p_{\alpha}^{0}$ in Eq. (5) was chosen to be one. For the two dimensional case, two simulation studies are discussed. In the first study, three interface energies $\gamma_{\alpha \beta}=\{0.1,1,2\}$ are chosen and the initial radii $r_{p}^{\text {init }}$ are varied. In the second study, for three initial radii $r_{p}^{\text {init }}=\{50,100,500\}$ the interface energies are changed in a more detailed range. The two studies are recorded in Fig. 2(a). The diagram shows the pressure difference over the inverse equilibrium radius. The analytic solutions are plotted as black lines and the simulation results are indicated by symbols. The first study corresponds to the three diagonal lines and the second study to three vertical lines. For three dimensional simulations, we analyzed three different initial radii as well as

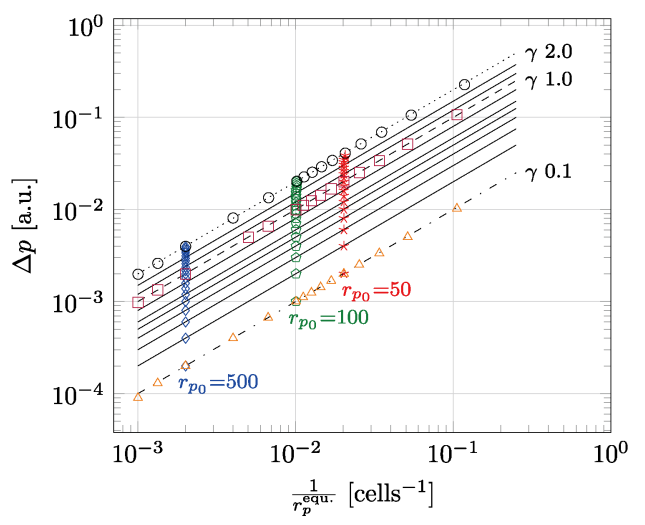

(a)

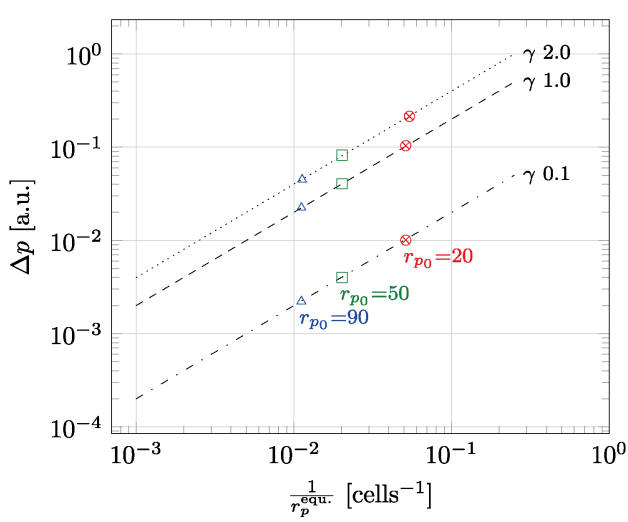

(b)

Fig. 2. Comparison of the simulated pressure difference with the Young-Laplace equation for different interface energies and pore diameter $r_{p}^{\text {equ }}$ for 2D in a) and 3D geometries in b). 


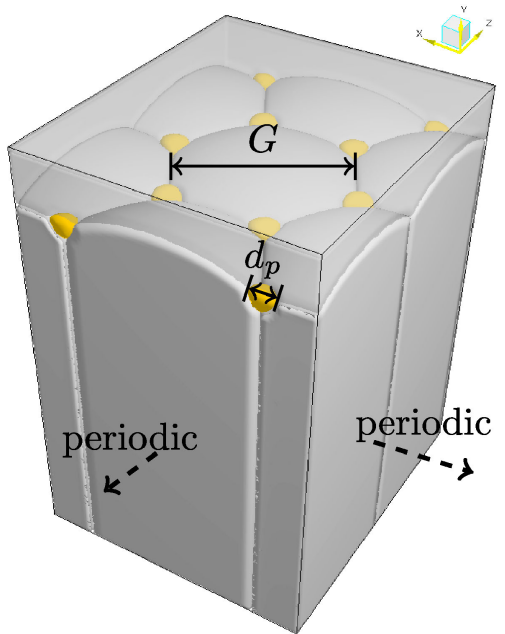

Fig. 3. Simulation setup and the varied parameters to study pore attachment and detachment.

three different interface energies as depicted in Fig. 2(b). The simulation results in Fig. 2 match the analytic solutions of Eq. (14) in both cases, 2D as well as 3D. Therefore, the introduced extension to the phase-field model is capable to capture the physical pressure behavior of the pores. Following, we study the pore-grain boundary interaction for different microstructural setups.

\subsection{Pores at grain boundaries of idealized geo- metries}

To study the behavior of pores on a moving grain boundary, we generate the grain geometry introduced by Miodownik et $\mathrm{al}^{26)}$ and modified by Tikare et al. ${ }^{8)}$ The geometry consists of hexagonal prisms with equal interface energies, resulting in a stable arrangement without grain growth among the hexagons. On top of this structure a single crystal is placed as depicted in Fig. 3. This geometry has the advantage of a constant driving force $F_{b}$ and hence a constant growth velocity for the single crystal into the hexagonal prisms. The velocity $v_{b}$ of the boundary without pores can be written as

$$
v_{b}=M_{b} F_{b}=\frac{2 \gamma_{\alpha \beta} M_{b}}{G}
$$

where $M_{b}$ is the mobility of the boundary between the hexagonal structure and the single crystal. $G$ denotes the diameter of the hexagon.

In the case of a pore-laden interface between the hexagonal prisms and the single crystal, the velocity can be written as

$$
v_{b}^{*}=M_{b}\left(F_{b}-N_{d} F_{p}\right)
$$

with $N_{d}$ as the pore density and $F_{p}$ the pore drag force. ${ }^{2), 27)}$ Tikare et al. ${ }^{8)}$ derived a more suitable description for the velocity of a pore-laden interface $v_{b}^{T}$ based on the models in $^{5), 28), 29)}$ where the pores are placed at the quadruple junctions. The velocity is written as

$$
v_{b}^{T}=F_{b} \frac{M_{p} M_{b}}{N_{A} M_{b}+M_{p}}
$$

with $M_{p}$ the mobility of the pore-grain interface and $N_{A}$ as the unit area of pores at the boundary. ${ }^{8)}$ The unit area of pores is defined as
Table 1. Simulation parameters for the hexagonal setup. The parameters in a) are used to study pore detachment and in b) to study pore-laden boundary migration

\begin{tabular}{ccll}
\hline \multicolumn{2}{c}{ parameter } & \multicolumn{2}{c}{ simulation value } \\
\cline { 3 - 4 } & & a) & b) \\
\hline$d_{p}$ & [cells] & 20 & $15,20,25,30$ \\
$G$ & [cells] & 150 & $100,200,250$ \\
$M_{p}$ & [a.u.] & 1 & $0.198,0.41,1,3.16$ \\
$M$ & [-] & $0.04,0.05$ & $5,10,15,20$ \\
\hline
\end{tabular}

$$
N_{A}=\frac{\pi N_{p}\left(\frac{d_{p}}{2}\right)^{2}}{A}
$$

with unit area $A$ and $N_{p}$ the number of pores therein.

The mobility of the pores can be derived by the flux of atoms. ${ }^{30)}$ For surface diffusion, the mobility $M_{p}$ can be written as

$$
M_{p}=\frac{D_{s} \delta_{s} \Omega}{\pi\left(\frac{d_{p}}{2}\right)^{4} k_{B} T} \propto \frac{1}{d_{p}^{4}}
$$

with the diffusion coefficient $D_{s}$, the thickness of the diffusion layer $\delta_{s}$, the atomic volume $\Omega$, the pore diameter $d_{p}$, the Boltzmann constant $k_{B}$ and the temperature $T$. As indicated, for a constant temperature, Eq. (19) depends on the size of the pore to the power four.

The grain structure consists of four hexagonal prisms, which are periodically continued according to symmetry. At the top and bottom of the domain, Neumann boundary conditions with a gradient of zero are imprinted. To study the interaction of pores and grain boundaries, the pore diameter $d_{p}$ as well as the hexagon diameter $G$ are varied. For the mobilities of the grain boundaries of the hexagonal prisms $M_{p c}$ and the single crystal $M_{s c}$ the same values are used, noted as $M_{b}$. Depending on the mobility ratio $M=M_{p} / M_{b}$ of the pore-grain boundary mobility and the graingrain boundary mobility, pore attachment is predicted for ratios larger than one and detachment for ratios smaller than one. ${ }^{27)}$ The simulations are carried out with a constant mobility ratio $M$ and a varying pore diameter. Note that the grain boundary mobility $M_{b}$ is not constant, since $M$ is kept constant and the mobility of the pore depends on their size according to Eq. (19). All interface energies $\gamma_{\alpha} \beta$ are chosen equal and are non-dimensionalized to the value one. The pore mobilities are calculated depending on their diameter $d_{p}$ following Eq. (19) and using constant values for $D_{s}$, $\delta_{s}, \Omega, k_{B}$ and $T$ for all simulations. This calculated mobilities are used as reciprocal values for the kinetic coefficient in Eq. (9). The parameters are summarized in Table 1.

The domain sizes for simulations with $G=100$ are $175 \times$ $250 \times 200$ voxel cells, for $G=150$ and 200 are $350 \times 250 \times$ 400 voxel cells and for $G=250$ are $440 \times 250 \times 500$ voxel cells. The simulations are conducted on the SuperMUC at the Leibniz-Rechenzentrum (LRZ) running about $8 \mathrm{~h}$ using 150, 576 and 1100 cores, respectively.

First we study the attachment and detachment behavior of the pores by using two different mobility ratios given in Table 1 a). Equal pores are placed at the quadruple junctions of the hexagonal prisms and the single crystal. Figure 4 displays the temporal evolution for the two mobility ratios of $M=0.04$ and $M=0.05$. For $M=0.05$, pore attachment at the grain boundary can be observed in Figs. 4(a) to 4(c). The pores remain at quadruple junctions over the time. For the lower ratio of $M=$ 0.04, depicted in Figs. 4(d) to 4(f), pore detachment can be 


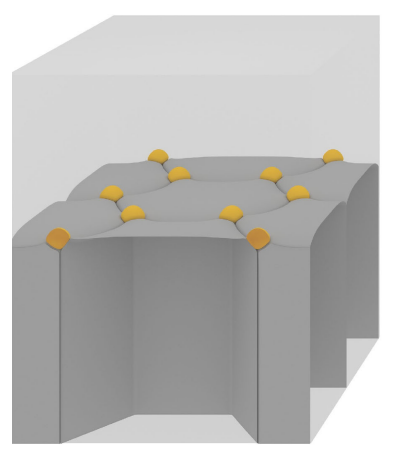

(a) $\mathrm{t}=13.36$

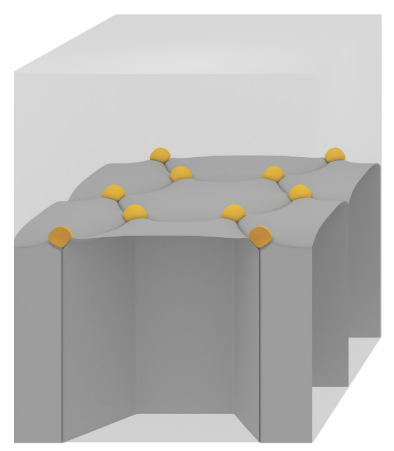

(d) $\mathrm{t}=13.36$

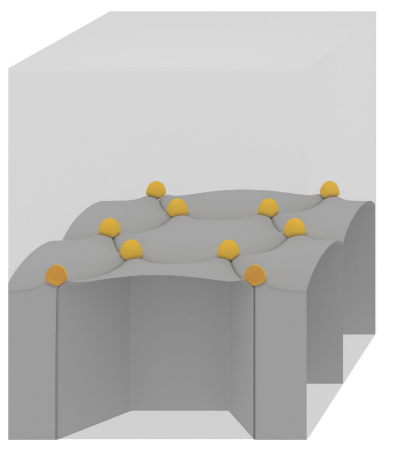

(b) $\mathrm{t}=300.6$

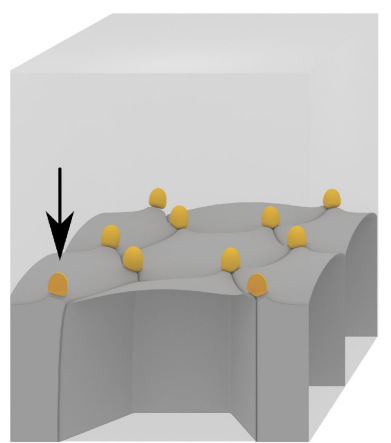

(e) $\mathrm{t}=300.6$

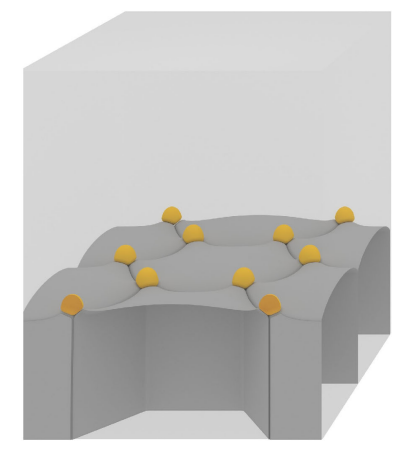

(c) $\mathrm{t}=484.3$

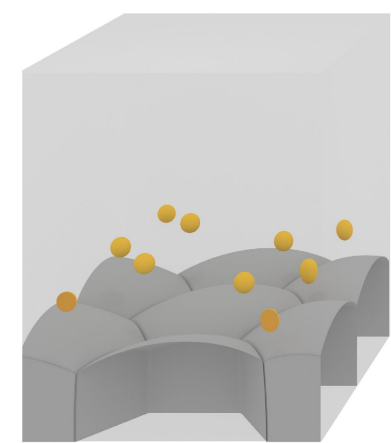

(f) $\mathrm{t}=484.3$

Fig. 4. Evolution of grain boundaries and pores in the case of pore attachment [Figs. 4(a) to 4(c)] with $M=0.05$ and of pore detachment [Figs. 4(d) to 4(f)] with $M=0.04$ for $G=150$ and for different time steps. The arrow indicates the moving pore position during detachment.

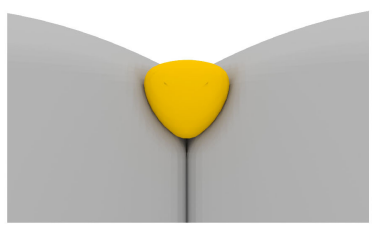

(a) pore at quadruple junc-
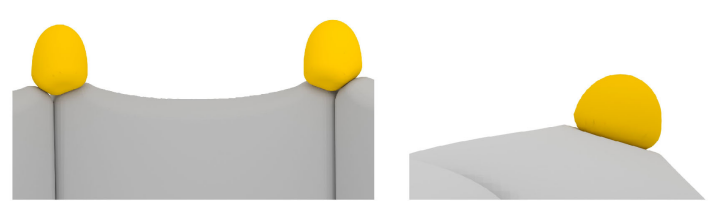

detachment

plane

Fig. 5. Locations and shapes of pores at grain boundaries.

observed. In both cases, the pores change their shape from a sphere to an ice cream cone shape as long as they are attached to the grain boundary, depicted in Fig. 5(a).

This shape was also observed by Tikare et al. ${ }^{8)}$ Before detachment, due to the higher velocity of the grain boundary plane, the pores are further deformed as shown in Fig. 5(b). Thereby the pores lose their stable position at the quadruple junctions as indicated by the arrow in Fig. 4(e). During the detachment, the pores shift from the quadruple point to a triple line and from there to a grain boundary depicted in Fig. 5(c), where they finally detach. For lower mobility ratios, the pores directly detach from quadruple points without changing their shape or position. To study the effect of the pore position, the pores are placed in the center of the hexagonal grains using the same parameters as before. The results are depicted in Fig. 6. In contrast to the results with pores at the grain junctions, both mobility ratios lead to a detachment of the pores, if they are located in the center of the hexagonal grains. In addition, the shape of the pores differs from those at quadruple junctions. They attain a shape similar to a droplet. For the lower mobility ratio, pores detach earlier.
To quantify the simulation results, we determine the velocity of pore drag effects (pore-laden and pore-free grain boundaries) and compare the results with analytic equations. We consider four mobility ratios, four pore sizes and three hexagon diameters as given in Table $1 \mathrm{~b}$ ). To prevent detachment of the pores, we choose higher mobility ratios. In Figs. 7(a) to 7(c) the velocity over the mobility ratio is depicted. The dashed line corresponds to the analytic solutions as given in Eq. (17). For all simulations, the measured velocity matches the analytic expression. The slightly higher velocities of grain boundaries in the simulations result from the assumed spherical shape of the pores for the analytic Eq. (17) and the resulting ice cream cone shape of a moving pore as shown in Fig. 5. This stretching of the pores in direction of movement leads to a smaller pore density compared to Eq. (18) and therefore to a higher velocity.

Similar findings are discussed by Tikare et al. ${ }^{8}$ using a MonteCarlo Potts model. For all hexagon diameters, the velocity decreases with an increasing pore diameter. As expected, larger mobility ratios as well as larger hexagons lead to a decrease of the velocity. 


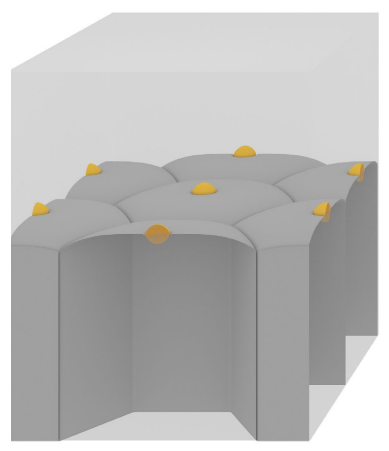

(a) $\mathrm{t}=13.36$

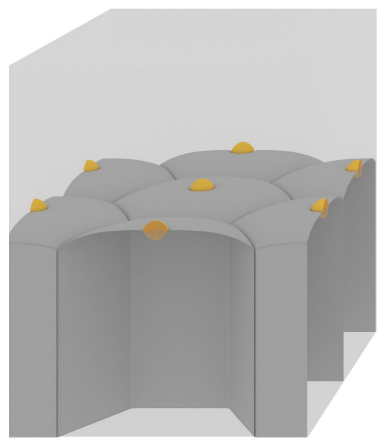

(d) $\mathrm{t}=13.36$

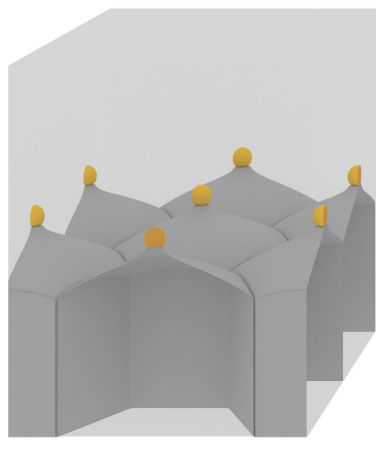

(b) $\mathrm{t}=167$

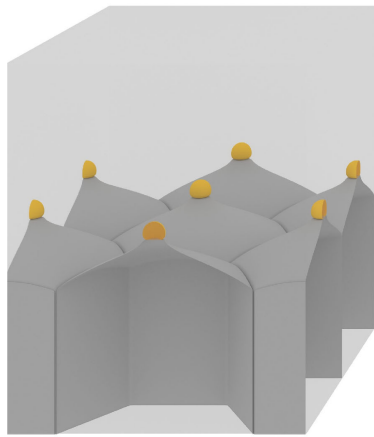

(e) $\mathrm{t}=116.9$

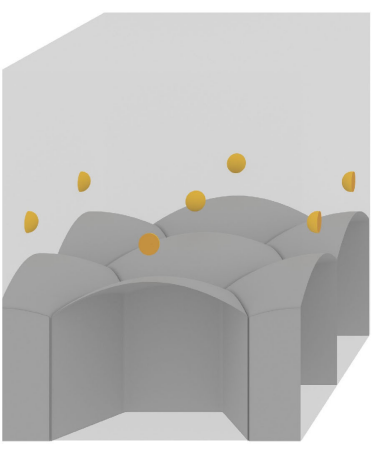

(c) $\mathrm{t}=217.1$

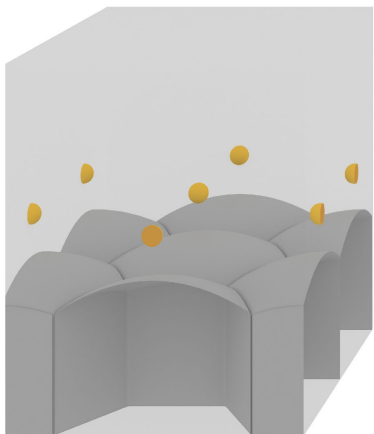

(f) $\mathrm{t}=183.7$

Fig. 6. Evolution of grain boundaries and pores in the center of the hexagonal grains with $M=0.05$ [Figs. 6 (a) to $6(\mathrm{c})]$ and with $M=0.04$ [Figs. $6(\mathrm{~d})$ to $6(\mathrm{f})$ ] for $G=150$ and for different time steps.

To validate general grain boundary motion, we additionally measure the velocities for a pore-free boundary and compare the values to the analytic Eq. (15). Four mobility ratios and a hexagon diameter of 200 cells are investigated. In Fig. 7(d) the velocities are plotted. Again, the measured values follow the analytic equation.

\subsection{Pores at grain boundaries of realistic geo- metries}

To study the pore-grain boundary interaction in more realistic geometries, the idealized hexagonal structure is replaced by a more realistic microstructure of multiple grains. This microstructure is generated by a Voronoi tessellation with a homogenous grain size distribution and a mean grain diameter $\bar{G} \cdot{ }^{13)}$ At the interface between the single crystal and the polycrystal, pore arrays with a defined spacing $f$ are placed. This is a common experimental setup to observe mobility induced effects such as pore drag. ${ }^{6), 31)-36)}$ In this setup, similar to the hexagonal structure, the polycrystal provides a driving force for the single crystal to grow into the polycrystal.

For realistic microstructures as in Fig. 8, pores can be located at quadruple junctions, triple junctions or grain boundaries. Additionally, the grains of the polycrystal coarsen and change the driving force with respect to the single crystal. Therefore the grain boundary velocity of the interface between the single crystal and the polycrystal is not constant.

To study the attachment and detachment of pores, we first derive an analytic expression for comparison with the simulation results. The driving force resulting from curvature of a large single grain growing into a polycrystal with the mean grain diameter $\bar{G}$ is estimated by a mean field approach as described by Hillert. ${ }^{38)}$ Based on this model, Rödel and Glaeser ${ }^{6)}$ assume the maximum driving Force $F_{b, \text { max }}$ to be

$$
F_{b, \max }=\frac{3 \gamma_{\alpha \beta}}{\bar{G}(t)}
$$

with the interface energy $\gamma_{\alpha \beta}$ of the grain boundaries in the polycrystal. The maximum velocity of the grain boundary can then be calculated as

$$
v_{b}=M_{b} F_{b, \max } .
$$

As the driving force depends on the grain size in the polycrystal and, thus, on the time $t$, a general grain growth law is used to determine $\bar{G}(t)$. The grain growth law of the polycrystal is formulated as

$$
\bar{G}(t)^{n}-\bar{G}(0)^{n}=k_{n} t
$$

with the time dependent grain size $\bar{G}(t)$, the initial grain size $\bar{G}(0)$, an empirical grain growth constant $k_{n}$ and the growth exponent $n$. In literature, different growth exponents are reported. Burke and Turnbull ${ }^{39)}$ describe the evolution of the mean grain size by a parabolic grain growth law with $n=2$.

To describe the growth of the single crystal into the polycrystal, Rödel and Glaeser ${ }^{6)}$ proposed a parabolic grain-growth law of the form

$$
\Delta L^{2}=k_{L} t
$$

with the growth constant $k_{L}$.

Using the root of Eq. (23) and the relation

$$
M_{b} \gamma_{\alpha \beta}=\frac{k_{L} \bar{G}(t)}{6 \Delta L}
$$

derived by Rödel and Glaeser ${ }^{37)}$ we can calculate the velocity of Eq. (21). The drag force of a pore can be written according to ${ }^{6), 27)}$ 


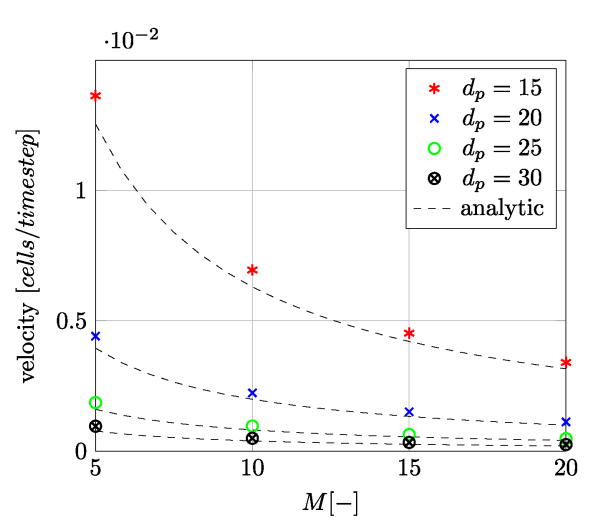

(a) $G=100$

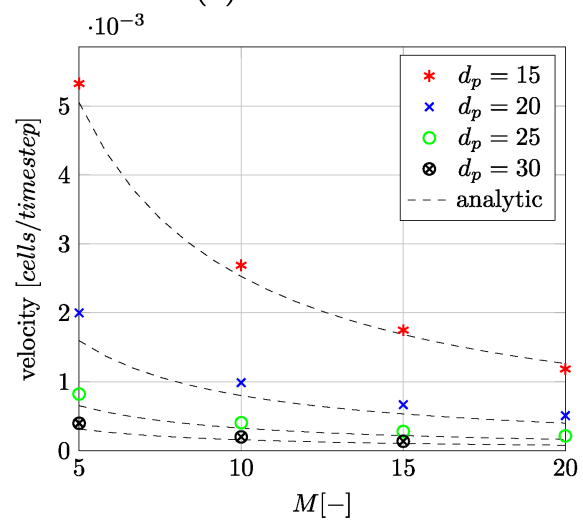

(c) $G=250$

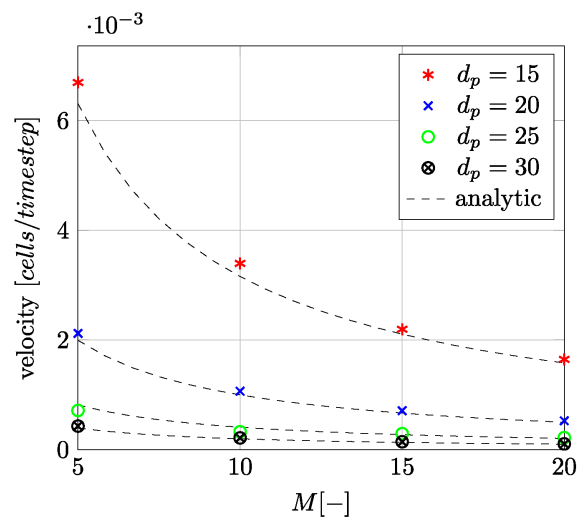

(b) $G=200$

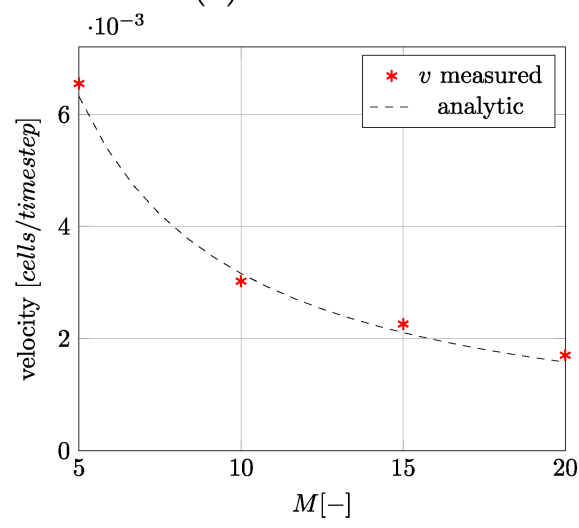

(d) $G=200$, without pores

Fig. 7. Comparison of computationally measured and analytically calculated velocities measured and calculated for the poreladen grain boundary for three grain sizes $G$ of the hexagonal grains and for varying mobilities $M$ and pore diameters $d_{p}$.

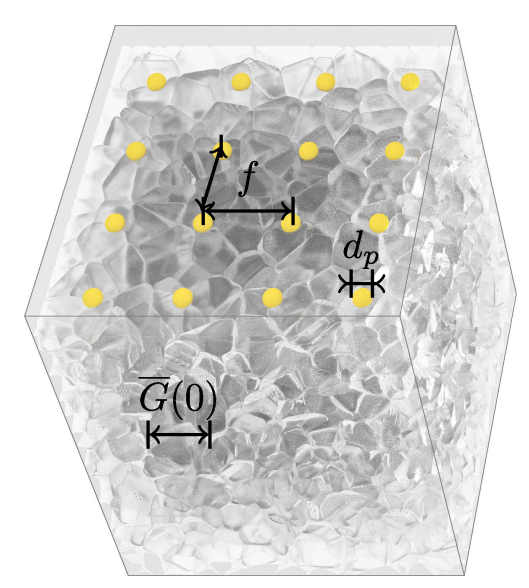

Fig. 8. Simulation setup and parameters related to the experiments of. ${ }^{37)}$ The pore spacing $f$, the pore diameter $d_{p}$ and the initial average grain diameter $\bar{G}(0)$ are illustrated.

as

$$
F_{p}=\pi \frac{d_{p}}{2} \gamma_{\alpha \beta}
$$

Inserting Eqs. (21) and (25) in Eq. (16), the critical grain size of the polycrystal for pore detachment is

$$
\bar{G}(t)<\frac{6 f^{2}}{\pi d_{p}}\left(1-\frac{v_{p}}{v_{b}}\right) .
$$

Table 2. Simulation parameter for realistic polycrystalline structures

\begin{tabular}{lll}
\hline \multicolumn{2}{c}{ parameter } & \multicolumn{1}{c}{ simulation value } \\
\hline$f$ & [cells] & $60,120,240$ \\
$\bar{G}(0)$ & [cells] & $15,20,30,35,40,45,50,55,60$ \\
$d_{p}$ & [cells] & $10,14,20$ \\
$M_{p}$ & [a.u.] & $0.12,0.49,1.87$ \\
$M$ & {$[-]$} & $1.6,0.42,0.1$ \\
\hline
\end{tabular}

With the relation Eq. (26) we can compare the pore detachment in realistic geometries.

For the simulations, we systematically vary the pore diameter $d_{p}$. The mobility of the grain boundaries $M_{b}$ is kept constant for all simulations. This results in a varying mobility ratio depending on the pore diameter in contrast to the previous studies where a constant ratio is established. The interface energy $\gamma_{\alpha \beta}$ of the grain-grain boundary is chosen as 0.5 and the interface energy of the pore-grain boundary as 1 . To study the effect of pore spacing $f$, we consider three pore diameters. Additionally, we systematically vary the initial mean grain size of the polycrystal for one selected and fixed pore diameter of $d_{p}=14$. The used parameters are given in Table 2.

The domain size for a pore spacing of $f=240$ cells is $480 \times$ $480 \times 300$ voxel cells and $240 \times 240 \times 300$ voxel cells for pore spacings of $f=60$ and 120 cells. All simulations are conducted on the SuperMUC at the Leibniz-Rechenzentrum (LRZ) in Munich using 2449 cores for $16 \mathrm{~h}$ for the large domain and 289 cores for $10 \mathrm{~h}$ for the smaller ones. 


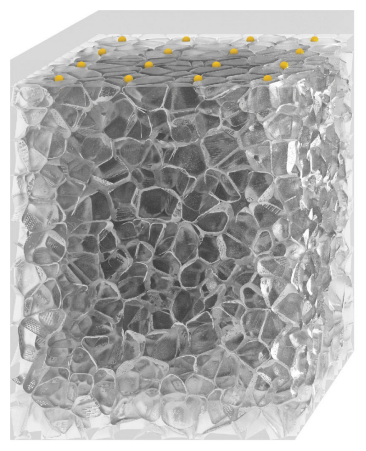

(a) initial filling

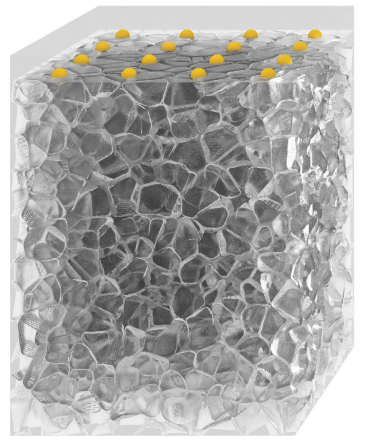

(d) initial filling

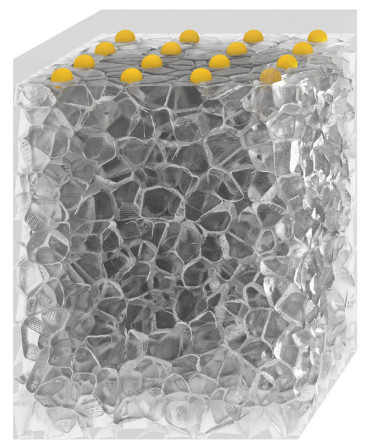

(g) initial filling

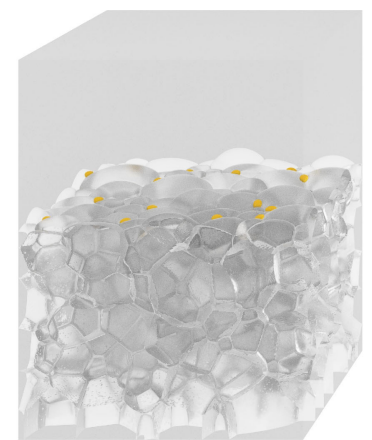

(b) $t=1680$

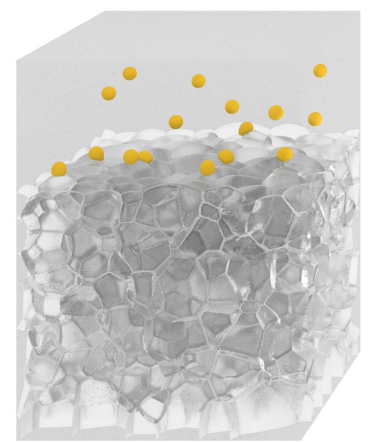

(e) $t=1260$

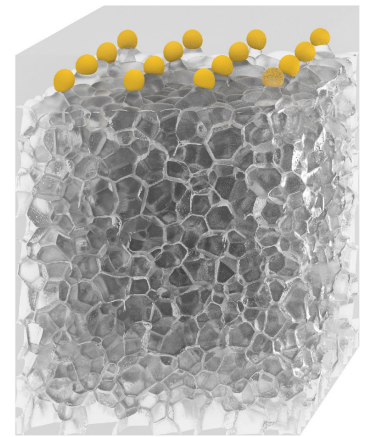

(h) $t=294$

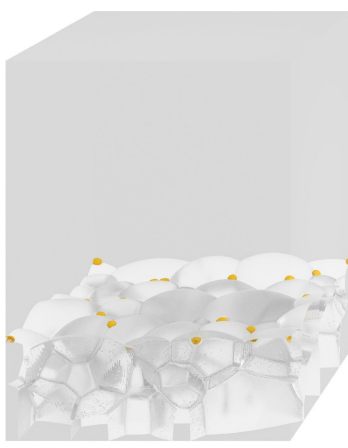

(c) $t=3780$

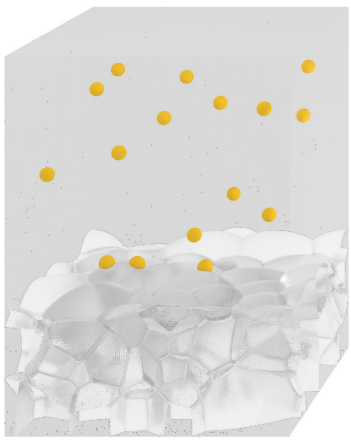

(f) $t=3360$

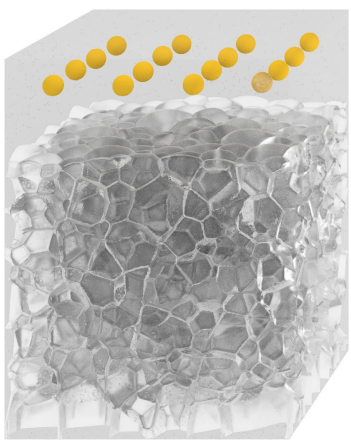

(i) $t=840$

Fig. 9. Evolution of grain boundaries and the pores in the case of pore attachment [Figs. 9 (a) to 9 (c)] with $d_{p}=10$, complete pore detachment [Figs. $9(\mathrm{~g})$ to 9 (i)] with $d_{p}=20$ and a partial state [Figs. 9(d) to 9(f)] with $d_{p}=14$ for different time steps. The domain size was $320 \times 240 \times 240$ voxel for all simulations.

First, the effect of the pore size $d_{p}$ on pore attachment and detachment is investigated. In Fig. 9, the evolution of the microstructure is given for different time steps and for three pore diameter of 10, 14 and 20 cells. Thereby three cases are observed, namely attachment, complete detachment and a partial detachment. This effects can also be seen in the Videos 1 and 2 youtube. (https://www.youtube.com/watch?v=YmHfn5Y6T7U, https:// www.youtube.com/watch?v=76A_Uwd1YqE)

For a diameter of $d_{p}=10$ cells [Figs. 9(a) to 9(c)], attachment of the pores occurs. During grain growth, pores migrate not only in growth direction of the single crystal, but also horizontal by following the triple and quadruple junctions of the polycrystal, as illustrated in Fig. 10. This effects are also visible in the videos on youtube. (https://www.youtube.com/watch?v=YmHfn5Y6T7U, https://www.youtube.com/watch?v=76A_Uwd1YqE)

In black circles the initial position of the pores, and in yellow the position at $t=1680$ is indicated. At the advanced time step,

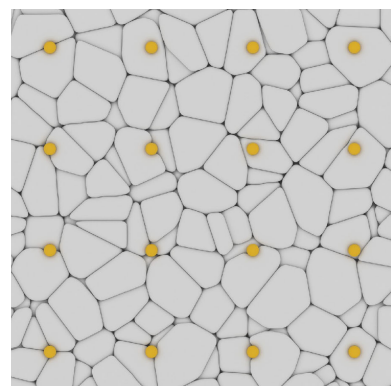

(a) $t=0$

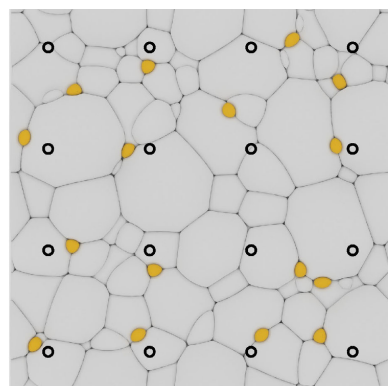

(b) $t=1680$
Fig. 10. Top view to indicate the pore movement for a pore diameter of $d_{p}=10, f=60$ and $\bar{G}(0)=20$. In green the initial position of the pores are indicated and in yellow the position at time $t=1680$. 


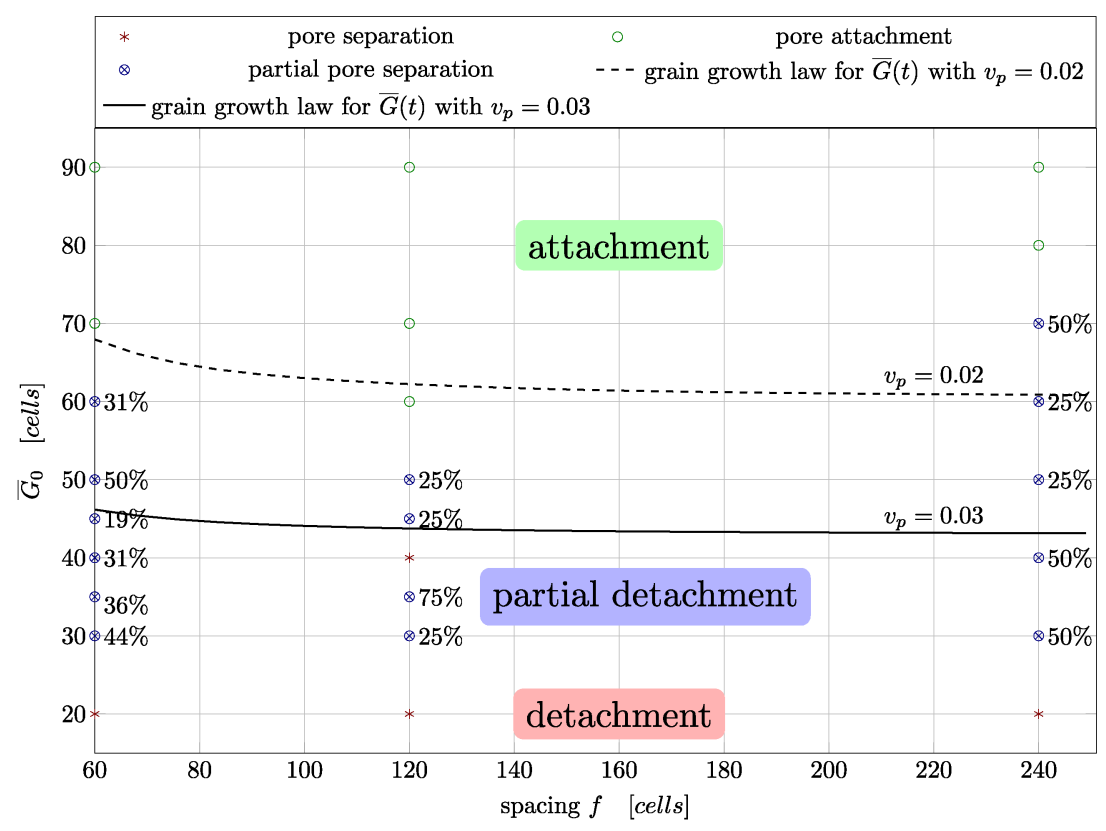

Fig. 11. Generated separation map for simulation results and comparison with the minimal and maximal pore velocities. The percentage numbers for the partial pore separation points indicate the fraction of detached pores.

all pores are located at triple and quadruple junctions.

For $d_{p}=14$ [Figs. 9(d) to 9(f)], a partial state can be detected with both, attached and detached pores. As found in the previous setting of hexagonal prisms, pore detachment depends on the location of the pores. For the partial state, pore detachment is only observed from grain boundary planes and not from triple or quadruple junctions.

For a pore diameter of $d_{p}=20$ [Figs. 9 (g) to 9(i)], instant pore detachment is observed. The pores do not change their position but remain at their initial position, which can be attributed to the low mobility ratio.

To further analyze the effects of partial detachment for a pore diameter of 14 cells, the grain size $\bar{G}(0)$ in the polycrystal is changed.

Figure 11 shows a map of the pore behavior in the simulations for different initial grain sizes $\bar{G}$ and pore spacings $f$. The red marks indicate simulations with complete pore detachment. Green marks show pore attachment and in blue, partial detachment with a particular percentage of detached pores is given.

To obtain the dashed and the solid line from Eq. (26), the maximum pore velocity is measured immediately before the detachment in the simulated microstructures. A lower and an upper pore velocity limit is found by exploiting the maximum velocity of the first and last event of pore detachment of the same pore array for each simulation. The averaged limits are found to be $v=0.02$ and $v=0.03$.

The morphology map in Fig. 11 indicates three regions, which is in good accordance to experimental work on alumina. ${ }^{6)}$ The lower part, corresponds to conditions for pore detachment given by Eq. (26) and grain sizes smaller 30 cells. In the upper part, for grain sizes larger 70 cells, pores stay attached to the grain boundary, due to low driving force resulting in a slow migration of the interface. In between, a diffuse region appears, in which partial detachment is observed. This region is close to the analytic solution for pore detachment Eq. (26).

In Fig. 11, the fraction of detached pores is given. One should expect that the fraction of detached pores increases with decreasing grain size of the polycrystal, since the driving force for migration and, thus, the migration rate increases. However, this trend is not clearly confirmed which might be explained by the limited statistics of the simulations in Fig. 11. Since our simulation setup uses only a limited number of pores (16 for a pore size of 60 cells and 4 for a pore size of greater than 120 cells), the initial pore position is statistically not well represented. For idealized hexagonal geometries, we showed that pore detachment is influenced by the location of the pore in the microstructure: pores at quadruple points tend to migrate first to triple lines and then to grain boundary planes, where they finally detach. Hence, we can conclude the initial location of a pore is important in the non-ideal polycrystalline microstructures: a pore initially located at a boundary plane will detach much easier than a pore at a triple line or quadruple line. The pores at the latter two positions first migrate along the boundary planes towards the center before detachment. This migration is highlighted in Fig. 10. Figure 10(a) displays the initial position of pores before grain growth, whereas Fig. 10(b) refers to an advanced grain coarsening state. The initial positions are added as black circles. The still attached pores in the final stage are colored yellow. Clearly a horizontal migration of pores can be observed. Simultaneously, grain growth occurs in the polycrystalline matrix leading to a decreasing driving force and hence a decreasing migration rate of the single crystal interface. If the horizontal movement is too slow, the migration rate of the interface decreases in the mean time and, eventually, becomes too low for pore detachment. Accordingly, the initial position of a pore has a strong influence on whether the pore detaches or not. Hence no clear trend in the fraction of detached pores can be observed in Fig. 11. Therefore, larger simulations are needed to reveal the statistical trend in pore detachment. However, the general behavior of pore detachment fits theory according to Eq. (26) and experimental results $\left.{ }^{6}\right)$ well.

Overall, the results presented above show that simulating pore attachment and detachment with the introduced phase-field model gives reasonable results. Accordingly, further studies should 
focus on the impact of attached pores during grain growth in final stage sintering. As stated in the introduction, grain growth occurs in final stage sintering, as soon as pores detach and grain boundary migration occurs. The present model can be used to reveal the complex behavior of grain growth and sintering and to complete the knowledge on grain growth in porous systems.

\section{Conclusion and outlook}

In this work we extend a phase-field model to study the pore drag effect. The pore stability is validated with the YoungLaplace equation. To study the behavior of pore attachment and detachment at grain boundaries, we consider an idealized hexagonal geometry and a realistic microstructure setup. For the idealized setup the simulation results match the analytic equations for the grain boundary velocity of pore-laden and porefree grain boundaries. For the realistic setup three cases are observed: Pore attachment, detachment and partial detachment, as previously found in experiments. ${ }^{6}$ ) To quantify the mechanisms and effects, a pore separation map is established in dependence of pore spacing and grain size reflecting the analytic predictions.

Our main conclusions are: (i) the introduced phase-field model agrees with the analytic solutions for pressure and grain boundary velocity. (ii) Detachment of pores depends on the initial location of the pores, e.g. grain boundary, triple and quadruple junction. (iii) Pores tend to migrate from quadruple junction to triple junctions to boundary planes, where they finally detach. (iv) The separation of pores depends on the velocity of grain-boundaries and hence grain size in the polycrystal. (v) For realistic microstructures, partial pore detachment can be observed, resulting from variance of the initial pore location. (vi) For realistic microstructures, the analytic solutions ignore the variance in pore location and hence give only a rough estimation for pore detachment. (vii) The pore spacing has a minor effect on the pore detachment.

To improve the understanding of the pore shape and location on the separation condition, further studies are necessary. To gain insight into more realistic setups comparable with experiments, we will study the effects of heterogeneous grain size distributions, non-uniform mobility distributions and anisotropic grain boundary properties in larger microstructures elements in forthcoming papers. Additionally advanced diffusion mechanisms will be considered and compared with the pressure model based on the ideal gas approach.

Acknowledgments We are grateful for the provided computational resources at the Leibniz Rechenzentrum in Munich (LRZ). We acknowledge Christopher Serr for helping to visualize the simulation data, Georges Barthelemy for preliminary tests and Frank Wendler for fundamental work on formulating the pressure driving force. We further thank the ministry MWK of the state Baden-Wuerttemberg for financial support through the cooperative graduated school "Gefügestrukturanalyse und Prozessbewertung".

\section{References}

1) J. Acker, H. Kungl, R. Schierholz, S. Wagner, R. A. Eichel and M. J. Hoffmann, J. Eur. Ceram. Soc., 34, 4213-4221 (2014).

2) R. J. Brook, J. Am. Ceram. Soc., 52, 56-57 (1969).

3) R. L. Coble, J. Appl. Phys., 32, 787-792 (1961).

4) S. J. Bennison and M. P. Harmer, J. Am. Ceram. Soc., 73, 833837 (1990).

5) G. Gottstein and L. Shvindlerman, Acta Metall. Mater., 41, 3267-3275 (1993).

6) J. Rödel and A. M. Glaeser, J. Am. Ceram. Soc., 73, 3302-
$3312(1990)$.

7) G. E. Moore, Proc. IEEE, 86, 82-85 (1998).

8) V. Tikare, M. Miodownik and E. Holm, J. Am. Ceram. Soc., 84, 1379-1385 (2001).

9) M. Selzer, Mechanische und Strömungsmechanische Topologieoptimierung mit der Phasenfeldmethode. PhD thesis (2014).

10) K. Ankit, B. Nestler, M. Selzer and M. Reichardt, Contributions to Mineralogy and Petrology, 166, 1709-1723 (2013).

11) K. Ankit, J. Urai and B. Nestler, J. Geophys. Res., B Solid Earth, 120, 3096-3118 (2015).

12) K. Ankit, M. Selzer, C. Hilgers and B. Nestler, Journal of Petroleum Science Research, 4, 79-96 (2015).

13) A. Vondrous, Grain growth behavior and efficient large scale simulations of recrystallization with the phase-field method. $\mathrm{PhD}$ thesis (2014).

14) X. N. Jing, J. H. Zhao, G. Subhash and X.-L. Gao, Mater. Sci. Eng., A, 412, 271-278 (2005).

15) Y. U. Wang, Acta Mater., 54, 953-961 (2006).

16) V. Kumar, Z. Z. Fang and P. C. Fife, Materials Science and Engineering: A, 528, 254-259 (2010).

17) K. Asp and J. Ågren, Acta Mater, 54, 1241-1248 (2006).

18) B. Nestler, H. Garcke and B. Stinner, Phys. Rev. E, 71, 041609 (2005).

19) A. Vondrous, B. Nestler, A. August, E. Wesner, A. Choudhury and J. Hötzer, "Metallic Foam Structures, Dendrites and Implementation Optimizations for Phase-Field Modeling", in High Performance Computing in Science and Engineering '11: Transactions of the High Performance Computing Center, Stuttgart (HLRS) 2011 (W. E. Nagel, D. H. Kröner and M. M. Resch, eds.), Springer, 2015 ed., 2011.

20) A. Vondrous, M. Selzer, J. Hötzer and B. Nestler, Int. J. High Perform. C., 28, 61-72 (2014).

21) J. Hötzer, A. Vondrous, J. Ettrich, M. Jainta, A. August, D. Studenvoll, M. Reichardt, M. Selzer and B. Nestler, "Phasefield simulations of largescale microstructures by integrated parallel algorithms", in High Performance Computing in Science and Engineering '14: Transactions of the High Performance Computing Center, Stuttgart (HLRS) 2014 (W. E. Nagel, D. H. Kröner and M. M. Resch, eds.), Springer, 2015 ed., 52015 .

22) J. Hötzer, M. Jainta, M. B. Said, P. Steinmetz, M. Berghoff and B. Nestler, "Application of large-scale phase-field simulations in the context of highperformance computing", in High Performance Computing in Science and Engineering '15: Transactions of the High Performance Computing Center, Stuttgart (HLRS) 2015 (W. E. Nagel, D. H. Kröner and M. M. Resch, eds.), Springer.

23) J. Hötzer, O. Tschukin, M. Ben Said, M. Berghoff, M. Jainta, G. Barthelemy, N. Smorchkov, D. Schneider, M. Selzer and B. Nestler, J. Mater. Sci., accepted. (2015).

24) N. Moelans, Acta Mater, 59, 1077-1086 (2011).

25) S. G. Kim, D. I. Kim, W. T. Kim and Y. B. Park, Phys. Rev. E, 74, 061605 (2006).

26) M. Miodownik, J. Martin and A. Cerezo, Philos. Mag. A, 79, 203-222 (1999).

27) M. N. Rahaman, Ceramic processing and sintering. New York: Taylor \& Francis, revised. ed., (2003).

28) J. Cahn, Acta Metall. Mater., 10, 789-798 (1962).

29) K. Lücke and H. Stüwe, Acta Metall. Mater., 19, 1087-1099 (1971).

30) S.-J. L. Kang, Sintering: Densification, Grain Growth and Microstructure. Butterworth-Heinemann, 1 ed., 2 (2005).

31) S.-M. An, B.-K. Yoon, S.-Y. Chung and S.-J. L. Kang, Acta Mater., 60, 4531-4539 (2012).

32) R. D. Monahan and J. W. Halloran, J. Am. Ceram. Soc., 62, 564-567 (1979).

33) Y. Finkelstein, S. M. Wiederhorn and B. J. Hockey, "Migration of sapphire interfaces into vitreous bonded aluminum oxide", Ceramic Transactions, 7 pp., vol. 258 (1990). 
34) R. Akiva, A. Katsman and W. D. Kaplan, J. Am. Ceram. Soc., 97, 1610-1618 (2014)

35) W. Rheinheimer, M. Bäurer, C. A. Handwerker, J. E. Blendell and M. J. Hoffmann, Acta Mater., 95, 111-123 (2015).

36) W. Rheinheimer, M. Bäurer and M. J. Hoffmann, Acta Mater., 101, 80-89 (2015).
37) J. Rödel and A. M. Glaeser, J. Am. Ceram. Soc., 73, 32923301 (1990).

38) M. Hillert, Acta Metall. Mater., 13, 227-238 (1965).

39) J. E. Burke and D. Turnbull, Prog. Met. Phys., 3, 220-292 (1952). 